\title{
Standard Errors of Correlations Adjusted for Incidental Selection
}

\author{
Nancy L. Allen \\ Educational Testing Service \\ Stephen B. Dunbar \\ University of lowa
}

The standard error of correlations that have been adjusted for selection with commonly used formulas developed by Pearson (1903) was investigated. The major purposes of the study were (1) to provide largesample approximations of the standard error of a correlation adjusted using the Pearson-Lawley three-variable correction formula; (2) to examine the standard errors of adjusted correlations under specific conditions; and (3) to compare various estimates of the standard errors under direct and indirect selection. Two theorybased large-sample estimates of the standard error of a correlation adjusted for indirect selection were developed using the delta method. These two estimates were compared to one another, to a bootstrap estimate, and to an empirical standard deviation of a se-

\begin{abstract}
ries of adjusted correlations generated in a simulation study. The simulation study manipulated factors defined by sample size, selection ratio, underlying population distribution, and population correlations in situations that satisfied the basic assumptions of the Pearson-Lawley procedures. The results indicated that the large-sample and bootstrap estimates were very similar when the sample size was 500 and, in most cases, the simpler of the two large-sample approximations appears to offer a reasonable estimate of the standard error of an adjusted correlation without resorting to complex, computer-intensive approaches. Index terms: correlation coefficients, missing data, Pearson-Lawley corrections, selection, standard errors of correlations, validity studies.
\end{abstract}

A recurring problem in educational research is how to take into account nonrandom selection that has restricted the range of the variables of interest in correlational analyses. This problem has important implications for policy and practice in education and testing (Linn, 1983). Several expressions (Pearson, 1903) presented in matrix notation by Lawley (1944) are commonly used in selection settings to adjust for samples chosen on the basis of a direct selection variable; they are based on what Little and Rubin (1987) called the assumption of ignorable nonresponse. Pearson derived these expressions from wellknown linear regression relationships.

One of these expressions estimates the correlation in a population before selection by adjusting a correlation from a sample selected directly on the basis of one of the variables of interest (two-variable case, direct or explicit selection). Another of the Pearson-Lawley formulas adjusts a correlation from a sample selected on the basis of a variable different from the two variables of interest to estimate a correlation in the population before selection (three-variable case, indirect or incidental selection). The

APPLIED PSYCHOLOGICAL MEASUREMENT

Vol. 14, No. 1, March 1990, pp. 83-94

(C) Copyright 1990 Applied Psychological Measurement Inc.

0146-6216/90/010083-12\$1.85 
formula for this adjustment is

$R_{x y}=\frac{r_{x y}+\left[\left(S_{z} / s_{z}\right)^{2}-1\right] r_{x z} r_{y z}}{\left\{\left[1+\left(\left(S_{z} / s_{z}\right)^{2}-1\right) r_{x z}^{2}\right]\left[1+\left(\left(S_{z} / s_{z}\right)^{2}-1\right) r_{y z}^{2}\right]\right\}^{1 / 2}}$

where $r$ and $s$ denote correlations and standard deviations (SDs) for the selected sample, and $R$ and $S$ denote correlations and SDs for the total population. Observations are selected if the value of the variable $z$ is smaller or larger than a criterion value, and the variables $x$ and $y$ are indirectly influenced by the selection. To the extent that $\left(S_{z} / s_{z}\right)^{2}$ is greater than 1 , which occurs under explicit selection on $z$, the Pearson adjustment will add an increment to the observed sample correlation between $x$ and $y$. That increment is also affected by $r_{x z}$ and $r_{y z}$. In practice, one of two situations occurs: Either full information about $z$ is obtained both before and after selection takes place, or the variance of $z$ before selection is known from previous situations and information about $z$ is obtained only after selection takes place.

The Pearson-Lawley relationships between correlations for a selected sample and correlations for the full population hold if two assumptions are met. The first assumption is that there is a linear relationship between the selection variable and the other variables of interest. The second assumption is homoscedasticity, that the variances of the indirectly selected variables and the covariances of those variables with other variables do not depend on the value of the selection variable. For the two-variable case, this means that the slopes of the regressions for the selected sample and for the full population are equal $\left(b_{x z}=B_{x z}\right)$ and that the SDS of the indirectly selected variable conditioned on the selection variable for the selected sample and for a random sample are equal $\left(s_{x . z}=S_{x . z}\right)$. For the three-variable case, the slopes of the regressions of the two indirectly selected variables for the selected sample and for the full population are not necessarily equal $\left(b_{x y} \neq B_{x y}\right)$.

Most of the previous research concerning these formulas has examined the bias of the adjusted correlations when the assumptions of the Pearson-Lawley formulas have not been met or when the selection process is poorly modeled by the formulas. Another area of research that is important for the appropriate use of these adjusted correlations, but which has received less attention, is the variability of these estimates.

Despite the importance of standard errors (SES) of correlations, relatively few estimates of the SES of correlations adjusted with the Pearson-Lawley formulas have been derived or examined. For the twovariable case, a large-sample estimate of the SE of an adjusted correlation was first derived by Kelley (1923). A similar estimate was later derived by Bobko and Rieck (1980).

The principal differences between the work of Kelley and the more recent work of Bobko and Rieck involve (1) the method used to derive the SE estimates, (2) the form of the estimate, and (3) the estimate of the SE of the unadjusted correlation used in the expression for the SE. An advantage of the delta method used by Bobko and Rieck to derive the SE estimate of an adjusted correlation is that asymptotic covariances of correlations required in more complicated cases involve only correlations with one variable in common. The estimate of the SE of the unadjusted correlation used by Bobko and Rieck is appropriate for bivariate normal or, at least, bivariate mesokurtic variables in the part of the population fitting the selection criterion. Kelley suggested the use of an "appropriate' estimate of the SE of an unadjusted correlation and provided an estimate dependent on the kurtoses of the distributions for the two variables of interest. This more general expression is appropriate if the relationship between the variables in the part of the population fitting the selection criterion is linear, as is true if the assumptions of the Pearson-Lawley adjustments are met.

Other studies of the SEs of correlations adjusted for range restriction include Gullickson and Hopkins (1976), Brandt, Mclaughlin, Wise, and Rossmeissl (1984), and Mendoza, Hart, and Powel (1987). Gullickson and Hopkins (1976) calculated empirical nomograms for confidence intervals of adjusted correlations for the two-variable case. Brandt et al. (1984) used a large dataset containing Armed Services Vocational Aptitude Battery scores and training grades to develop bootstrap estimates of the SES of 
adjusted correlations. They intended these to be useful descriptive statistics in situations where the PearsonLawley adjusted correlations were to be reported as part of a criterion-related validity study. Mendoza et al. (1987) developed empirical confidence intervals for the correlation adjusted using the two-variable formula when the Pearson-Lawley assumptions were violated.

SEs of correlations adjusted for the more complex selection situations, including the three-variable case, have not been investigated thoroughly. Accordingly, the primary purpose of this study was to investigate SE estimates for correlation coefficients adjusted using the Pearson and Lawley methods. Specifically, this investigation had three major purposes: (1) to provide large-sample approximations of the SE of a correlation adjusted using the Pearson-Lawley three-variable correction formula; (2) to examine the SES of adjusted correlations under specific conditions; and (3) to compare various estimates of the SEs under direct and indirect selection.

\section{Method}

\section{SE Estimates for the Three-Variable Case}

Two theory-based large-sample estimates of the SE of a correlation adjusted for indirect selection were developed using the delta method (based on Taylor series expansions for variances, and on formulas for the asymptotic variances and covariances of correlations). As assumed by Kelley and by Bobko and Rieck, $S_{z} / S_{z}$ was considered fixed in this application of the delta method. In the simulation study that follows, however, $S_{z} / s_{z}$ was obtained for each replication independently. The use of the delta method in this setting was appropriate because each unadjusted sample correlation had a variance of order $N^{-1}$ and the formula for the three-variable adjustment was differentiable at the selected population correlations. Both SE estimates found using the delta method are of the form

$$
\begin{aligned}
\operatorname{SE}\left(R_{x y}\right)= & {\left[\left(R_{x y}^{\prime}\right)^{2} \operatorname{Var}\left(r_{x y}\right)+\left(R_{x z}^{\prime}\right)^{2} \operatorname{Var}\left(r_{x z}\right)+\left(R_{y z}^{\prime}\right)^{2} \operatorname{Var}\left(r_{y z}\right)\right.} \\
& \left.+2 R_{x y}^{\prime} R_{x z}^{\prime} \operatorname{Cov}\left(r_{x y}, r_{x z}\right)+2 R_{x y}^{\prime} R_{y z}^{\prime} \operatorname{Cov}\left(r_{x y}, r_{y z}\right)+2 R_{x z}^{\prime} R_{y z}^{\prime} \operatorname{Cov}\left(r_{x z}, r_{y z}\right)\right]^{1 / 2}
\end{aligned}
$$

where, for example, $R_{x z}^{\prime}$ is the derivative of the adjusted correlation $R_{x y}$ with respect to the unadjusted correlation $r_{x z}$. These derivatives are shown in Table 1.

The difference between the two estimates of the SE of the adjusted correlation is in the estimates chosen for the variances and covariances. Table 2 contains two sets of estimates for the necessary asymptotic variances and covariances of the unadjusted correlations. The estimates are explained in more detail in Allen (1987/1988) and the assumptions for each estimate are given by Isserlis (1916). The

\section{Table 1}

Partial Derivatives of the Adjusted Correlation $R_{x y}$ With Respect to Each Unadjusted Correlation

\begin{tabular}{ccc}
\hline $\begin{array}{c}\text { Partial } \\
\text { Derivative }\end{array}$ & $\begin{array}{c}\text { Unadjusted } \\
\text { Correlation }\end{array}$ & $\begin{array}{c}\text { Derivative of } R_{x y} \text { Evaluated at } \\
\text { Estimates of the Parameters* }\end{array}$ \\
\hline$R_{x y}^{\prime}$ & $r_{x y}$ & {$\left[1+W\left(r_{x z}\right)^{2}\right]^{-1 / 2}\left[1+W\left(r_{y z}\right)^{2}\right]^{-1 / 2}$} \\
$R_{x z}^{\prime}$ & $r_{x z}$ & $W\left[1+W\left(r_{x z}\right)^{2}\right]^{-3 / 2}\left[1+W\left(r_{y z}\right)^{2}\right]^{-1 / 2}\left[r_{y z}-r_{x y} r_{x z}\right]$ \\
$R_{y z}^{\prime}$ & $r_{y z}$ & $W\left[1+W\left(r_{x z}\right)^{2}\right]^{-1 / 2}\left[1+W\left(r_{y z}\right)^{2}\right]^{-3 / 2}\left[r_{x z}-r_{x y} r_{y z}\right]$ \\
\hline *Where $W=\left(S_{z} / s_{z}\right)^{2}-1$ &
\end{tabular}


Table 2

Estimated Sampling Variances and Covariances of Unadjusted Correlations

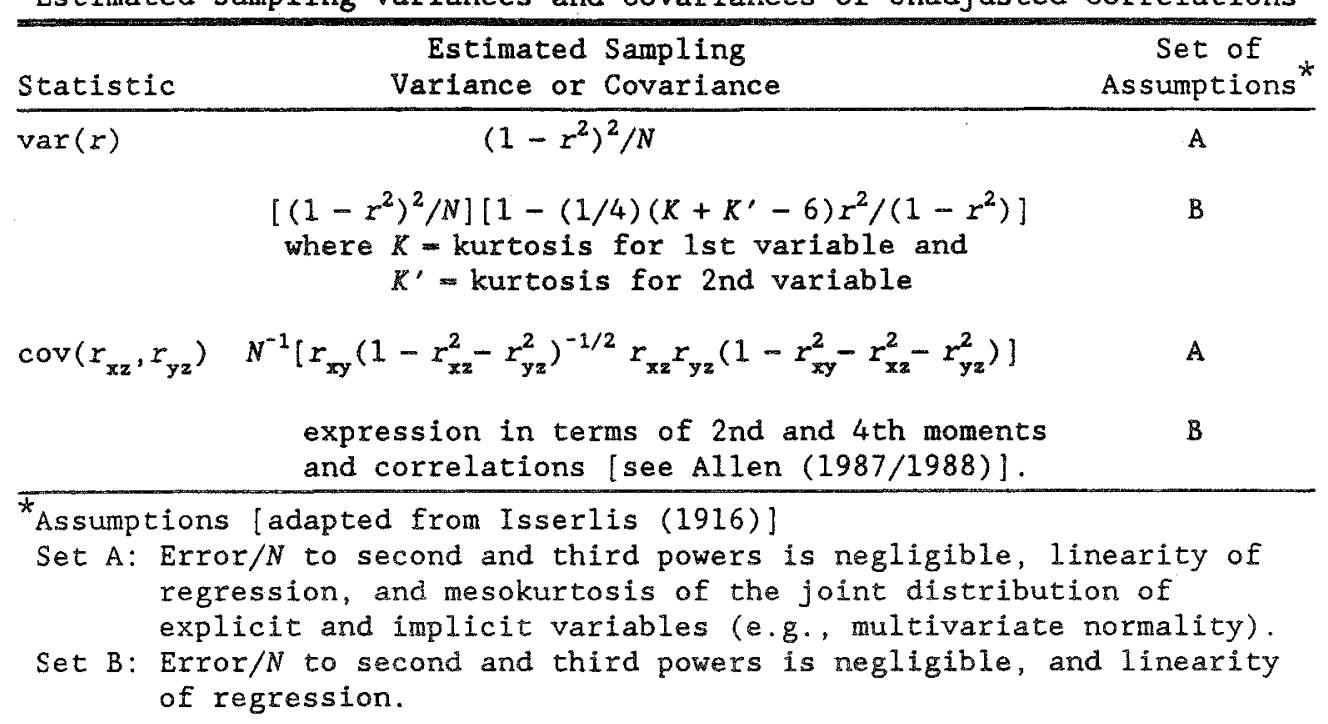

variance and covariance estimates fitting the Set $A$ assumptions correspond to the estimate of the variance of the unadjusted correlation used by Bobko and Rieck. The assumptions of this first estimate of the SE of the adjusted correlation probably are not strictly met in real settings involving nonrandom selection. The variance and covariance estimates fitting the Set B assumptions correspond to the estimate of the variance of the unadjusted correlation suggested by the work of Kelley. The more general assumptions of this second estimate of the SE of the adjusted correlation are more likely to be met than are the assumptions of the first estimate. However, the kurtoses of the distributions of the variables of interest may be difficult to estimate, hence there was some interest in assessing whether a practical difference existed.

These two large-sample estimates of the SE of an adjusted correlation in the three-variable case are complicated. In particular, if normality or at least mesokurtosis of the selected sample is not assumed, higher-order moments are needed to estimate the SE of an adjusted correlation. However, neither of these estimates requires sophisticated optimization routines or is computer intensive. Modern computer capabilities make all of these calculations possible and convenient.

\section{Simulation Study}

Comparisons. For the three-variable case, these two estimates were compared to one another, to a bootstrap estimate, and to an empirical SD of a series of 250 adjusted correlations generated in a simulation study. The bootstrap estimate was calculated for each sample from the data in 100 bootstrap samples of size $N$. Each bootstrap sample contained observations selected with replacement from the original sample. For each bootstrap sample, the unadjusted sample correlations, the SD of $Z$ after selection, and the value of $S_{z}$ were used to find the adjusted correlation. The bootstrap estimate was the SD of the 100 adjusted correlations from the bootstrap samples associated with each dataset.

Because the data generation procedure made it convenient, estimates of the SE of adjusted correlations were also compared for the two-variable case. These two-variable estimates were Bobko and Rieck's 
estimate, Kelley's estimate, a bootstrap estimate (again based on 100 bootstrap samples), and an empirical SD. All estimates were examined in the metric of the correlation.

Design. The simulation study manipulated factors defined by (1) distributional characteristics (multivariate normal, multivariate platykurtic), (2) population correlations, (3) selection ratio, and (4) size of the selected sample, in situations that satisfied the basic assumptions of linearity and homoscedasticity. The data for both the three-variable and two-variable situations were taken from the same samples, generated to fit trivariate distributions. The samples were chosen from six populations. Three of these populations had multivariate normal distributions, while three had multivariate platykurtic distributions. A standardized beta $(2,2)$ distribution was chosen as the basis for the platykurtic populations because the kurtosis for this distribution, 2.14, was reasonably close to the median kurtosis of 2.39 for actual achievement test data found by Brandenburg (1972/1973). The beta distribution is also curtailed at both ends of the distribution in a way not unlike distributions of test scores.

Three population correlation matrices $\left(P_{1}, P_{2}, P_{3}\right)$, shown in Table 3 , were used to generate the data from the two types of distributions. For the three-variable case, the population correlation values that were estimated were $.25, .50$, and .75 , while for the two-variable case, the estimated population values were $.20, .50$, and .80 . Each of the datasets contained approximately $10 \%, 50 \%$, or $90 \%$ of the original simulated observations and 50,100, or 500 selected observations. The above set of conditions represented 54 combinations of the four design parameters included in the simulation study, and each set of conditions was replicated 250 times. As stated above, each of the datasets fit the assumptions required for use of the Pearson-Lawley formulas.

\section{Results}

The computer simulation contributed new information about the SES of adjusted correlations in three areas; examples of this information are presented in the following tables. Tables presenting the average estimates of the SES of the adjusted correlation for the three-variable case and for the two-variable case under all 54 experimental conditions are in the appendix, as are tables of the means of the unadjusted and adjusted sample correlations.

\section{Comparisons of the SE Estimates}

A comparison was made of different estimates of the SES of adjusted correlations. For the threevariable case, the bootstrap estimate and the two large-sample estimates provided in this study were

Table 3

Unselected Population Correlation Matrices for Populations

$P_{1}, P_{2}, P_{3}$, where $Z$ = the Explicit Selection Variable,

$X={ }^{2}$ Incidental Selection Variable (e.g., Test Score), and $Y$ an Incidental Selection Variable

(e.g., Criterion Variable)

\begin{tabular}{|c|c|c|c|c|c|c|c|c|c|}
\hline & \multicolumn{3}{|c|}{$P_{1}$} & \multicolumn{3}{|c|}{$P_{2}$} & \multicolumn{3}{|c|}{$P_{3}$} \\
\hline & $Z$ & $X$ & $Y$ & 2 & $X$ & $\bar{Y}$ & $\bar{Z}$ & $\bar{X}$ & $\bar{Y}$ \\
\hline$z$ & 1.00 & 20 & 30 & 1.00 & .50 & .80 & 1.00 & .80 & .95 \\
\hline$Y$ & & 1.00 & .25 & & 1.00 & .50 & & 1.00 & .75 \\
\hline$X$ & & & 1.00 & & & 1.00 & & & 1.00 \\
\hline
\end{tabular}


compared. In general, the averages for the three estimates were close to one another and to the empirical SD of the 250 adjusted correlations for each set of conditions when the sample size was 500 . They were also relatively close to one another when the sample size was 100 and the selection ratio was .5 or .9 .

A specific example of this can be seen in Table 4 for samples from the population with the platykurtic distribution and the first correlation matrix. In this case for three variables, the full population correlation to be estimated was .25 . When the size of the selected sample was 500 , the averages of the 250 values of the three types of estimates for the SE were the same regardless of the selection ratio. These averages were generally the same as the empirical SD of the 250 adjusted correlations, the exception being when the selection ratio was only .1. When the sample size was 100 , the averages of the SE estimates were within .01 of one another. For instance, for selection ratio .1, the averages were $.22, .23$, and .23 . These averages differed from the empirical SD of .18 more than the averages for selection ratios .5 and .9 differed from the empirical SDs of .10 and .09 , respectively. In this example, when the sample size was 50 and the selection ratios were .5 and .9 , the averages of the SE estimates and the empirical SDS were close to one another in value. When the sample size was 50 and the selection ratio was .1 , larger differences between the average SE estimates and the empirical SD occurred. In general, more differences between average SE estimates and the empirical SD occurred when the sample size was 50 , particularly for small selection ratios. For the two-variable case, the bootstrap estimate and the large-sample estimates were also close to one another and to the empirical SD when the sample size was 500 and, in most cases, when the sample size was 100 .

\section{Magnitudes of the SEs}

Information about the magnitudes of the SEs of adjusted correlations is available for the three-variable case. Examples of some of the relationships that hold can be seen in Table 4. Several of these relationships are expected on the basis of theory and intuition. For instance, given other conditions, smaller SES of the

Table 4

Average Estimates of Standard Errors of the Adjusted Correlation Between $X$ and $Y$ in the Three-Variable Case With Underlying Platykurtic Distribution for Population Matrix $P_{1}$ (.25 Was Estimated)

\begin{tabular}{|c|c|c|c|c|}
\hline \multirow[b]{2}{*}{$N$} & \multirow[b]{2}{*}{$\begin{array}{l}\text { Empirical } \\
\text { SD }\end{array}$} & \multicolumn{3}{|c|}{ Standard Errors } \\
\hline & & Bootstrap & $\begin{array}{c}\text { First } \\
\text { Large-Sample }\end{array}$ & $\begin{array}{c}\text { Second } \\
\text { Large-Sample }\end{array}$ \\
\hline \multicolumn{5}{|c|}{ Selection Ratio $=.1$} \\
\hline 50 & .27 & .31 & .33 & .33 \\
\hline 100 & .18 & .22 & .23 & .23 \\
\hline 500 & .07 & .08 & .08 & .08 \\
\hline \multicolumn{5}{|c|}{ Selection Ratio $=.5$} \\
\hline 50 & .15 & .15 & .15 & .15 \\
\hline 100 & 10 & .10 & .11 & .11 \\
\hline 500 & .05 & .05 & .05 & .05 \\
\hline \multicolumn{5}{|c|}{ Selection Ratio $=.9$} \\
\hline 50 & .14 & .13 & .13 & .14 \\
\hline 100 & .09 & .09 & .09 & .10 \\
\hline 500 & .04 & .04 & .04 & .04 \\
\hline
\end{tabular}


adjusted correlation were associated with larger sample sizes and with larger selection ratios (percentages of selected observations).

For the two large-sample estimates, the relationship between SEs and sample size is to be expected because the formulas contain a factor of $N^{-1 / 2}$. It is of interest that a factor of $N^{-1 / 2}$ also appeared to account for differences among empirical SDS and for differences among the bootstrap SEs. In other words, the relationship between the SES and $N$ is as expected.

Comparisons across selection ratios. An examination of the SEs for different selection ratios shows that differences between the SEs for selection ratios .1 and .5 were much larger than the differences between the SEs for selection ratios .5 and .9 , particularly for the underlying beta distribution. Small values of the ratio of the SDs of the selection variable before and after selection $\left(S_{z} / s_{z}\right)$ occurred when the percentage of observations selected was large. Therefore, small SEs of the adjusted correlation correspond to small values of $S_{z} / s_{z}$. In fact, the differences between $S_{z} / s_{z}$ for selection ratios .1 and .5 were much larger than the differences between $S_{z} / s_{z}$ for selection ratios .5 and .9 . The differences between SES for different selection ratios can also be seen in Table 5, which contains the empirical SDs for the threevariable case when the size of the selected sample was 500, although differences in the SES were small.

Comparisons across population correlation matrices. Relationships between the population correlation matrix and the magnitude of the $\mathrm{SE}$, and between the underlying distribution and the magnitude of the SE, can also be seen in Table 5. In general, given other conditions, smaller SEs of adjusted correlations correspond to the estimation of larger population correlations. This was absolutely true for the two-variable case. For the three-variable case, however, the relationship between the three population correlations also contributed to the size of the SES. The effects of stringent selection in the platykurtic case and the interplay between correlations in the population correlation matrix $P_{2}$ are exemplified by the unusually large SD for the three-variable case when the selection ratio was .1 (.11). Differences between the two underlying distributions are further examined below.

Table 5

Standard Deviations of 250 Adjusted Correlations Between

$X$ and $Y$ for the Two- and Three-Variable Cases $(N=500)$

\begin{tabular}{lllll}
\hline \hline & $\begin{array}{c}\text { Population } \\
\text { Correlation }\end{array}$ & \multicolumn{3}{c}{ Selection Ratio } \\
\cline { 5 - 5 } & & & .5 & .9 \\
\hline $\begin{array}{l}\text { Three-Variable Case } \\
\text { Normal Distribution }\end{array}$ & .25 & .05 & .05 & .04 \\
$P_{1}$ & .50 & .05 & .04 & .04 \\
$P_{2}$ & .75 & .03 & .02 & .02 \\
$P_{3}$ & & & & \\
Platykurtic Distribution & .25 & .07 & .05 & .04 \\
$P_{1}$ & .50 & .11 & .05 & .03 \\
$P_{2}$ & .75 & .05 & .02 & .02 \\
$P_{3}$ & & & & \\
Two-Variable Case & & & & \\
Normal Distribution & .20 & .10 & .07 & .05 \\
$P_{1}$ & .50 & .07 & .05 & .04 \\
$P_{2}$ & .80 & .03 & .02 & .02 \\
$P_{3}$ & & & & \\
Platykurtic Distribution & .20 & .19 & .08 & .05 \\
$P_{1}$ & .50 & .14 & .05 & .04 \\
$P_{2}$ & .80 & .05 & .02 & .01 \\
$P_{3}$ & & & & \\
\hline
\end{tabular}


Comparisons between the two-and three-variable cases. Table 5 also shows that although the relationships between averages of the SE estimates and factors in the simulation design were similar for the two-variable case, the relationships were stronger for the two-variable case. In fact, when the correlation being estimated was the same (.50), the magnitudes of the SEs of the adjusted correlation for the threevariable case were smaller than the magnitudes for the two-variable case. This appears to be primarily due to the additional information available at the extremes of the bivariate distribution of the two indirectly selected variables for the three-variable case. In summary, given other conditions, smaller SEs were associated with smaller values of $S_{z} / s_{z}$, with larger sample sizes, with larger selection ratios, with larger underlying correlations (for the two-variable case), and with incidental rather than explicit selection.

\section{Comparisons Between Platykurtic and Normal Underlying Distributions}

Information was obtained about the SE of adjusted correlations for datasets selected from a platykurtic population. In general, the relationships between design characteristics and the magnitudes of the SES for datasets associated with the beta distribution were similar to those associated with the normal distribution. Specifically, expected relationships between the magnitude of the SE and sample size, population correlations, and selection ratios were observed. Although relationships could be identified for each underlying distribution, the role of $S_{z} / s_{z}$ was clearer when SEs for the two types of distributions could be compared. As stated previously, the magnitude of $S_{z} / s_{z}$ is small for large selection ratios.

This can be seen in Table 6 in terms of the population value $\Sigma_{z} / \sigma_{z}$ that is estimated by $S_{z} / s_{z}$ for the two types of distributions. The difference between magnitudes of $\Sigma_{z} / \sigma_{z}$ for the two different distributions of the selection variable can also be seen in Table 6 . When selection is not stringent, $\Sigma_{z} / \sigma_{z}$ values for the normal distribution and for the beta distribution are close in magnitude. However, when the selection ratio is .1 , the value of $\Sigma_{z} / \sigma_{z}$ for the beta distribution is almost twice as large as the value for the normal distribution. As expected, the values in Table 5 show that the corresponding empirical SDS of the adjusted correlation for the normal and platykurtic distributions are similar in value for selection ratios .5 and .9 . However, for the selection ratio .1, the SDs of the adjusted correlation for the platykurtic distribution are noticeably larger in value than the SDS of the adjusted correlation for the normal distribution.

To the extent that test scores are curtailed and platykurtic, as are the observations from the beta $(2,2)$ distribution, concern over SES of adjusted correlations is heightened. However, contrary to the statement by Lord and Novick $\left(1968\right.$, p. 147) that a value of $\Sigma_{z} / \sigma_{z}$ larger than 1.4 is severe enough to cause concern in using the Pearson-Lawley adjustments, the SDs in Table 5 indicate that when the sample size is large the SDS are reasonably small in relation to the size of the correlation being estimated, even when selection is more stringent than $70 \%$ selection. If concern about the use of the Pearson-Lawley adjustments when $\Sigma_{z} / \sigma_{z}$ is larger than 1.4 is well-founded, it must be based, as Lord and Novick alluded, on the size of the bias of the adjustments when the assumptions are not strictly met.

Table 6

Ratio of the Standard Deviations of the Selection

Variable in the Full Population and in the Population Fitting the Selection Criterion $\left(\Sigma_{z} / \sigma_{z}\right)$

\begin{tabular}{|c|c|c|c|}
\hline \multirow{2}{*}{$\begin{array}{l}\text { Distribution } \\
\text { of the } \\
\text { Selection Variable }\end{array}$} & \multicolumn{3}{|c|}{ Selection Ratio } \\
\hline & .1 & .5 & .9 \\
\hline $\begin{array}{l}\text { Normal }(0,1) \\
\text { Standardized Beta }(2,2)\end{array}$ & $\begin{array}{l}2.43 \\
4.73 \\
\end{array}$ & $\begin{array}{l}1.66 \\
1.84 \\
\end{array}$ & $\begin{array}{l}1.19 \\
1.14 \\
\end{array}$ \\
\hline
\end{tabular}




\section{Summary}

A comparison of the SE estimates indicated that, except for the case of very strict selection, the estimates were similar to one another and to the empirical SDS. SES were smallest when $S_{z} / s_{z}$ was small, when $N$ was large, when selection was not strict, when selection was incidental rather than explicit, and (for the two-variable case) when the population correlation that was being estimated was large. For the three-variable case, the SE depended not only on the correlation that was being estimated, but also on the other two population correlations. Finally, underlying distributions similar to those underlying test scores appear to contribute to SEs larger than those that occur when the underlying distribution is normal.

\section{Conclusions}

When validity evidence is provided for tests used to select applicants, or for tests administered to examinees selected for some other reason, correlations are often used. On the basis of this study, two suggestions can be made to practitioners using the Pearson-Lawley adjustments in these settings. The first is a recommendation for the use of the estimates for the standard error of the adjusted correlations. The second is a warning concerning the use of an adjusted correlation without reporting an estimate of its SE, and is based on the magnitude of the standard error in a variety of settings, including those for the platykurtic distribution and for the three-variable case. Because these suggestions are made in the context of an exact fit of the data to the Pearson-Lawley assumptions of linearity and homogeneity of variance, they indicate "best case" results for real data that may not fit the assumptions exactly.

Comparisons of the alternative estimates of standard errors led to one dominant finding: For settings permitting some degree of confidence in the Pearson-Lawley adjustments, the SE estimates provided very similar assessments of the degree of sampling error. Given these results, the use of the simpler of the two large-sample estimates is suggested for both the two- and three-variable cases when the sample size is large. Although the assumptions for this estimate may not be strictly true (the multivariate distribution underlying unadjusted correlations is not usually mesokurtic), this approximation appeared to offer a reasonable estimate of the SE of an adjusted correlation without resorting to the computer-intensive approach of the bootstrap estimate or to the complexity of the large-sample estimate with less stringent assumptions.

An examination of the magnitudes of the estimates for the SE of the three-variable adjusted correlations indicated that even when the sample size was 100 , adjusted correlations were relatively variable when the selection ratio was .1. The empirical SD of the 250 adjusted correlations for the normal datasets with a population correlation of .50 , for instance, was .13. For the set of conditions described above, the empirical SD was .26 when the underlying distribution was platykurtic. The SE of an adjusted correlation was always large relative to the correlation itself when the selection ratio was .1, but particularly when the underlying distribution was platykurtic, when the population correlations were small, or when the sample size was less than 500. For the two-variable case, an examination of the relative magnitudes of the SDs showed that caution in the use of the adjusted correlation as a simple point-estimate for small selection ratios was also in order. 


\begin{tabular}{|c|c|c|c|c|c|c|c|c|c|c|c|c|c|}
\hline $\begin{array}{c}\text { Aver } \\
\text { for } \\
\text { R }\end{array}$ & $\begin{array}{l}\text { age } \\
\text { the } \\
\text { eplic } \\
\text { Le }\end{array}$ & $\begin{array}{l}\text { tes } \\
\text { and } \\
s \text { in } \\
\text { amp } 1\end{array}$ & $\begin{array}{c}\text { of Sta } \\
\text { Three } \\
\text { Each } \\
\text { e Est }\end{array}$ & $\begin{array}{l}\text { andar } \\
\text {-Vari. } \\
\text { Cell, } \\
\text { imate, }\end{array}$ & $\begin{array}{l}\text { d Err } \\
\text { able } \\
\text { BTs } \\
\text { anc }\end{array}$ & $\begin{array}{l}\text { le } \\
\text { th } \\
\text { S } \\
\text { is } \\
\text { is }\end{array}$ & $\begin{array}{l}\text { Adju } \\
\text { is } t \\
\text { ootst } \\
\text { he Se } \\
\end{array}$ & $\begin{array}{l}\text { ted } \\
\text { e St } \\
\text { ap Es } \\
\text { ond I }\end{array}$ & $\begin{array}{l}\text { Corre } \\
\text { andar } \\
\text { stima } \\
\text { Large }\end{array}$ & $\begin{array}{l}\text { Bet } \\
\text { Latio } \\
\text { SE1 i } \\
\text { Le Es }\end{array}$ & $\begin{array}{l}\text { ween } \\
n \text { of } \\
s \text { the } \\
\text { timat }\end{array}$ & $\begin{array}{l}X \text { and } \\
\text { the } 25 \\
\text { First }\end{array}$ & $\begin{array}{l}Y \\
50 \\
t\end{array}$ \\
\hline & & & & & & & Lecti & n Rat & Eio & & & & \\
\hline Popula- & & & 1 & & & & & & & & 9 & 9 & \\
\hline tion & $N$ & SD & BTSE & LSE1 & LSE2 & $S D$ & BTSE & LSE1 & LSE2 & $S D$ & BTSE & LSE1 & LSE2 \\
\hline $\begin{array}{l}\text { Two-Var } \\
\text { Norma }\end{array}$ & $\begin{array}{l}\text { iable } \\
1 \text { Dis }\end{array}$ & Eion & & & & & & & & & & & \\
\hline$P_{1}$ & 50 & .32 & .28 & .29 & .29 & .22 & .21 & .21 & 21 & .16 & .15 & .16 & .16 \\
\hline & 100 & .21 & .22 & .22 & .22 & .14 & .15 & .15 & .15 & .11 & .11 & .11 & .11 \\
\hline & 500 & .10 & .10 & .10 & .10 & .07 & .07 & .07 & .07 & .05 & .05 & .05 & .05 \\
\hline$P_{2}$ & 50 & .23 & .23 & .23 & .23 & .18 & .16 & .16 & .16 & .13 & .12 & .12 & .12 \\
\hline & 100 & .16 & .16 & .16 & .16 & .11 & .12 & .12 & .12 & .08 & .08 & .08 & .08 \\
\hline & 500 & .07 & .07 & .07 & .07 & .05 & .05 & .05 & .05 & .04 & .04 & .04 & .04 \\
\hline$P_{3}$ & 50 & .10 & .11 & .10 & .09 & .07 & .07 & .07 & .07 & .06 & .05 & .06 & .06 \\
\hline & 100 & .07 & .07 & .07 & .06 & .05 & .04 & .05 & .04 & .04 & .03 & .04 & .04 \\
\hline & 500 & .03 & .03 & .03 & .03 & .02 & .02 & .02 & .02 & .02 & .02 & .02 & .02 \\
\hline Beta D & Distr & ion & & & & & & & & & & & \\
\hline$P_{1}$ & 50 & .50 & .41 & .45 & .45 & .23 & .22 & .23 & .23 & .16 & .15 & .15 & .15 \\
\hline & 100 & .40 & .33 & .36 & .36 & 18 & .16 & .17 & .17 & .11 & .11 & .12 & .12 \\
\hline & 500 & .19 & .18 & .19 & .19 & .08 & .08 & .08 & .08 & .05 & .05 & .05 & .05 \\
\hline$P_{2}$ & 50 & .46 & .37 & .40 & .40 & .17 & .17 & .17 & .18 & .11 & .11 & .12 & .12 \\
\hline & 100 & .34 & .30 & .31 & .31 & .13 & .12 & .13 & .13 & .08 & .08 & .08 & .09 \\
\hline & 500 & .14 & .14 & .14 & .14 & .05 & .05 & .06 & .06 & .04 & .04 & .04 & .04 \\
\hline$P_{3}$ & 50 & .26 & .24 & .20 & .20 & .07 & .07 & .07 & .08 & .05 & .04 & .05 & .07 \\
\hline 3 & 100 & .13 & .16 & .13 & .13 & .04 & .05 & .05 & .05 & .03 & .03 & .04 & .05 \\
\hline & 500 & .05 & .05 & .05 & .05 & .02 & .02 & .02 & .02 & .01 & .01 & .02 & .02 \\
\hline Three-Va & ariab & ase & & & & & & & & & & & \\
\hline Normal & 1 Dis & utior & & & & & & & & & & & \\
\hline$P_{1}$ & 50 & .18 & .19 & .19 & 19 & .15 & .15 & .15 & .15 & .14 & .13 & .13 & .13 \\
\hline & 100 & .11 & .12 & .12 & .12 & 10 & .10 & .10 & .10 & .10 & .09 & .10 & .10 \\
\hline & 500 & .05 & .05 & .05 & .05 & .05 & .05 & .05 & .05 & .04 & .04 & .04 & .04 \\
\hline$P_{2}$ & 50 & .18 & .19 & .19 & .19 & .14 & .14 & .14 & .14 & .12 & .11 & .11 & .11 \\
\hline & 100 & .13 & .13 & .13 & .13 & 10 & .10 & .10 & .10 & .08 & .08 & .08 & .08 \\
\hline & 500 & .05 & .06 & .06 & .06 & .04 & .04 & .05 & .04 & .04 & .03 & .04 & .04 \\
\hline$P_{3}$ & 50 & .10 & .11 & .10 & .09 & .08 & .07 & .08 & .07 & .07 & .06 & .07 & .07 \\
\hline & 100 & .07 & .07 & .07 & .06 & .05 & .05 & .05 & .05 & .05 & .04 & .05 & .05 \\
\hline & 500 & .03 & .03 & .03 & .03 & .02 & .02 & .02 & .02 & .02 & .02 & .02 & .02 \\
\hline Beta & Distr & ion & & & & & & & & & & & \\
\hline$P_{1}$ & 50 & .27 & .31 & .33 & .33 & .15 & .15 & .15 & .15 & .14 & .13 & .13 & .14 \\
\hline & 100 & .18 & .22 & .23 & .23 & .10 & .10 & .11 & .11 & .09 & .09 & .09 & .10 \\
\hline & 500 & .07 & .08 & .08 & .08 & .05 & .05 & .05 & .05 & .04 & .04 & .04 & .04 \\
\hline$P_{2}$ & 50 & .36 & .30 & .32 & .32 & .15 & .15 & .15 & .15 & .11 & .11 & .11 & .12 \\
\hline & 100 & .26 & .23 & .24 & .24 & .11 & .11 & .11 & .11 & .08 & .07 & .08 & .08 \\
\hline & 500 & .11 & .11 & .11 & .11 & .05 & .05 & .05 & .05 & .03 & .03 & .04 & .04 \\
\hline$P_{3}$ & 50 & .25 & .23 & .20 & .20 & .08 & .07 & .08 & .09 & .06 & .05 & .07 & .07 \\
\hline & 100 & .12 & .15 & .13 & .13 & .05 & .05 & .06 & .06 & .04 & .04 & .05 & .05 \\
\hline & 500 & .05 & .05 & .05 & .05 & .02 & .02 & .02 & .03 & .02 & .02 & .02 & .02 \\
\hline
\end{tabular}


Table B

Unselected Population and Average Sample

Correlations Between $X$ and $Y$ for the Two- and

Three-Variable Cases: $\rho_{x y}$ is the Unselected Population

Correlation, $r_{x y}$ is the Unadjusted Sample Correlation, and $R_{x y}$ is the Adjusted Sample Correlation

\begin{tabular}{llll} 
Popula- & \multicolumn{3}{c}{ Selection Ratio } \\
\cline { 4 - 6 } & $N$ & $\rho_{x y}$ & $\frac{1}{r_{x y} R_{x y}} \frac{5}{r_{x y} R_{x y}} \frac{.9}{r_{x y} R_{x y}}$ \\
\hline
\end{tabular}

Two-Variable Case

Normal Distribution

$\begin{array}{lll}P_{1} & 50 \quad .20\end{array}$

$100-20$

$500 \quad .20$

$P_{2} \quad 50 \quad .50$

$100 \quad .50$

$500 \quad .50$

$\begin{array}{lrl}P_{3} & 50 & .80\end{array}$ $\begin{array}{ll}100 & .80 \\ 500 & .80\end{array}$

$\begin{array}{llll}.08 & .16 & .12 & .19\end{array}$

$.18 \quad .20$

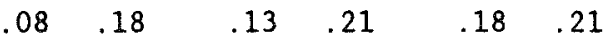

$.08 \quad .20$

$\begin{array}{ll}.13 & .21 \\ .12 & .19\end{array}$

$.18 \quad .21$

$.24 \quad .48$

$.33 \quad .48$

$.43 \quad .49$

$.23 \quad .49$

$.32 \quad .48$

$.45 \quad .51$

.23 .50

$\begin{array}{ll}.33 & .48 \\ .62 & .70\end{array}$

$.44 \quad .50$

$.48 \quad .79$

$.62 \quad .79$

$.74 \quad .79$

$\begin{array}{llllll}.48 & .79 & .62 & .80 & .74 & .79\end{array}$

Beta Distribution

$\begin{array}{lrr}P_{1} & 50 & .20 \\ & 100 & .20\end{array}$

$.48 \quad .80$

$.63 \quad .80$

$.75 \quad .80$

500

.20

$.04 \quad .13$

.11 .19

$.18 \quad .20$

$P_{2} \quad 50$

.20

$.05 \quad .18$

$.11 \quad .18$

$.17 \quad .19$

$.05 \quad .20$

$.11 \quad .20$

$.18 \quad .20$

.11 .35

$.31 \quad .49$

$.45 \quad .50$

500

.50

$.12 \quad .41$

$.30 \quad .49$

$.45 \quad .50$

$P_{3} \quad 50$

$\begin{array}{llll}.12 & .48 & .30 & .50\end{array}$

$.58 \quad .79$

$.45 \quad .50$

$.27 \quad .73$

$.59 \quad .80$

$.76 \quad .80$

100

.80

$\begin{array}{rr}.27 & .77 \\ 26 & .79\end{array}$

$.59 \quad .80$

$.76 \quad .80$

Three-Variable Case

Normal Distribution

\begin{tabular}{|c|c|c|c|c|c|c|c|c|}
\hline \multirow{3}{*}{$P_{1}$} & 50 & .25 & .20 & .22 & .22 & .24 & .24 & .25 \\
\hline & 100 & .25 & .21 & .24 & .23 & .26 & .24 & .25 \\
\hline & 500 & .25 & .22 & .25 & .22 & .25 & .24 & .26 \\
\hline \multirow{3}{*}{$P_{2}$} & 50 & .50 & .27 & .47 & .35 & .48 & .45 & .50 \\
\hline & 100 & .50 & .28 & .49 & .34 & .48 & .45 & .51 \\
\hline & 500 & .50 & .28 & .50 & .35 & .50 & .44 & .50 \\
\hline \multirow{3}{*}{$P_{3}$} & 50 & .75 & .35 & .74 & .52 & .74 & .68 & .74 \\
\hline & 100 & .75 & .34 & .74 & .53 & .75 & .67 & .74 \\
\hline & 500 & .75 & .35 & .75 & .53 & .75 & .68 & .75 \\
\hline \multicolumn{9}{|c|}{ Distribution } \\
\hline \multirow{3}{*}{$P_{I}$} & 50 & .25 & .21 & .23 & .23 & .26 & .23 & .24 \\
\hline & 100 & .25 & .20 & .24 & .22 & .25 & .24 & .25 \\
\hline & 500 & .25 & .21 & .25 & .21 & .24 & .24 & .25 \\
\hline \multirow{3}{*}{$P_{2}$} & 50 & .50 & .22 & .37 & .33 & .49 & .46 & .50 \\
\hline & 100 & .50 & .21 & .41 & .32 & .49 & .45 & .50 \\
\hline & 500 & .50 & .22 & .48 & .33 & .50 & .46 & .50 \\
\hline \multirow{3}{*}{$P_{3}$} & 50 & .75 & .11 & .68 & .48 & .74 & .69 & .75 \\
\hline & 100 & .75 & .10 & .72 & .48 & .75 & .70 & .75 \\
\hline & 500 & .75 & .10 & .74 & .48 & .75 & .70 & .75 \\
\hline
\end{tabular}

Downloaded from the Digital Conservancy at the University of Minnesota, http://purl.umn.edu/93227.

May be reproduced with no cost by students and faculty for academic use. Non-academic reproduction requires payment of royalties through the Copyright Clearance Center, http://www.copyright.com/ 


\section{References}

Allen, N. L. (1988). An evaluation of standard errors for correlation coefficients adjusted for explicit and incidental selection (Doctoral dissertation, University of Iowa, 1987). Dissertation Abstracts International, $48,10 \mathrm{~A}$.

Bobko, P., \& Rieck, A. (1980). Large sample estimators for standard errors of functions of correlation coefficients. Applied Psychological Measurement, 4, 385398.

Brandenburg, D. C. (1973). The use of multiple matrix sampling in approximating an entire empirical norms distribution (Doctoral dissertation, University of Iowa, 1972). Dissertation Abstracts International, 33, 3368A.

Brandt, D., McLaughlin, D., Wise, L., \& Rossmeissl, P. (1984). Complex cross-validation of the validity of a predictor battery. Paper presented at the meeting of the American Psychological Association, Toronto.

Gullickson, A., \& Hopkins, K. (1976). Interval estimation of correlation coefficients corrected for restriction of range. Educational and Psychological Measurement, 36, 9-25.

Isserlis, L. (1916). On certain probable errors and correlation coefficients of multiple frequency distributions with skew regression. Biometrika, $11,185-190$.

Kelley, T. L. (1923). Statistical method. New York: Macmillan.

Lawley, D. N. (1944). A note on Karl Pearson's selection formulae. Royal Society of Edinburgh: Proceedings, Section A, 62, 28-30.
Linn, R. L. (1983). Pearson selection formulas: Implications for studies of predictive bias and estimates of educational effects in selected samples. Journal of Educational Measurement, 20, 1-15.

Little, R. J. A., \& Rubin, D. B. (1987). Statistical analysis with missing data. New York: Wiley.

Lord, F. M., \& Novick, M. R. (1968). Statistical theories of mental test scores. Reading MA: AddisonWesley.

Mendoza, J. L., Hart, D., \& Powel, A. (1987). Empirical confidence intervals for validity coefficient under range restriction: An application of the bootstrap. Paper presented at the meeting of the American Educational Research Association, Washington DC.

Pearson, K. (1903). Mathematical contributions to the theory of evolution-XI. On the influence of natural selection on the variability and correlation of organs. Philosophical Transactions of the Royal Society of London: Series A, 200, 1-66.

\section{Author's Address}

Send requests for reprints or further information to Nancy L. Allen, Educational Testing Service, 02-T, Rosedale Road, Princeton NI 08541, U.S.A. 